\title{
Dynamic Pattern Detection with Temporal Consistency and Connectivity Constraints
}

\author{
SKYLER SPEAKMAN \\ School of Computer Science, Carnegie Mellon University \\ speakman@cmu.edu
}

\begin{abstract}
This work explores scalable and accurate dynamic pattern detection methods in graph-based data sets. The proposed Dynamic Subset Scan method is applied to the task of detecting, tracking, and source-tracing contaminant plumes spreading through a water distribution system equipped with noisy, binary sensors. While static patterns affect the same subset of data over a period of time, dynamic patterns may affect different subsets of the data at each time step. These dynamic patterns require a new approach to define and optimize penalized likelihood ratio statistics in the subset scan framework, as well as new computational techniques that scale to large, real-world networks. To address the first concern, this work develops a new subset scan methods that allows the detected subset of nodes to change over time, while incorporating temporal consistency constraints to reward patterns that do not dramatically change between adjacent time steps. Second, the Additive GraphScan algorithm allows this novel scan statistic to process small graphs (500 nodes) in 4.1 seconds on average while maintaining an approximation ratio over $98 \%$ compared to an exact optimization method, and to scale to large graphs with over 12,000 nodes in 30 minutes on average. Evaluation results across multiple detection, tracking, and source-tracing tasks demonstrate substantial performance gains achieved by the Dynamic Subset Scan approach.
\end{abstract}

\section{Introduction}

Detecting patterns in massive data sets has multiple real-world applications in fields such as public health, law enforcement, and security. The "subset scan" approach to pattern detection treats the problem as a search over subsets of data, with the goal of finding anomalous subsets. This approach poses two main challenges: appropriately evaluating the "anomalousness" of a given subset, and the computational issue of searching through the exponentially many subsets of the data. Previous approaches $[8,12,10,11]$ have addressed the first concern by "scoring" each subset using likelihood ratio statistics such as the expectation-based Poisson (EBP) [12, 10] or expectation-based binomial (EBB) [8] scan statistics. This current work allows for a more sophisticated scoring function by penalizing the likelihood ratio statistic, considering additional prior information from each data element. The penalized likelihood ratio typically does not satisfy useful properties such as "linear-time subset scanning" [11], making efficient optimization over subsets a challenging task. However, the EBB likelihood ratio statistic can be written as an additive function, enabling efficient optimization of a penalized version of that statistic over subgraphs. The first major contribution of this work is the development of temporal consistency constraints which allow for additional penalties or rewards to act on the scoring function, rewarding spatial subsets that are temporally consistent with each other, and efficient optimization of the resulting, penalized scan statistic to detect dynamic clusters subject to these constraints. The 
second major contribution is the Additive GraphScan algorithm, which efficiently identifies anomalous (high scoring), connected subgraphs, thus incorporating both (hard) connectivity constraints and (soft) temporal consistency constraints. Each of these contributions will be discussed in detail below.

Many complex data sets containing emerging events or patterns are commonly represented in a known and fixed graph structure. Examples of this include water pipelines, transportation routes, power grids, and supply chains in general. While other recent work $[9,5]$ has focused on learning graph structure, here we assume a given graph structure and wish to detect which nodes are currently affected, by observing data produced at the nodes of the graph on each time step.

The motivating example comes from the field of public health: with focus on detecting, tracking, and source-tracing contaminant plumes in a water distribution system. Creating sensor networks for detecting deliberate or accidental contamination of these systems has been a popular research domain following the terror attacks of September 11, 2001. The "Battle of the Water Sensor Networks" (BWSN) [1] provided real-world data to teams tasked with placing perfect sensors to quickly detect contaminants and limit the amount of contaminated water consumed by the population. The placement problem is an interesting one explored further in [3, 7]. This current work focuses on the complementary problem of fusing data collected from noisy sensors assuming a given placement. Sensor fusion attempts to combine data from multiple distributed sensors in order to increase the detection power of the entire network [14].

The simulation proceeds by modeling simple, binary sensors at each pipe junction (graph node) in the system with a fixed false positive rate (e.g., FPR $=0.1$ ) and true positive rate (e.g., $\mathrm{TPR}=0.9$ ). An additional assumption is that each sensor operates independently of the others in the network. The simulations use the network structure and plumes provided in the BWSN data to generate sensor readings over the course of 12 one-hour intervals.

The task is then: Given (1) the graph structure (pipe network), (2) false positive and true positive rates of the sensors, and (3) independent observations from the sensors over time, the method(s) must provide: (A) whether or not a contaminant is present in the system for each onehour time step, (B) hour-to-hour tracking of which pipe nodes have been affected by the plume on the current and recent past time steps, and (C) source-tracing to determine which node(s) in the system spawned the contaminant. Corresponding evaluation metrics include: (A) average time (in hours) to plume detection as a function of false positive rate (number of false alarms per month), (B) spatial-temporal overlap coefficient between the true and detected subsets of nodes over time, and (C) spatial overlap coefficient between the true and identified subsets of source nodes.

This is not the first work to apply spatial or subset scan statistics to contamination early warning systems. Koch and McKenna [6] used Kulldorff's spatial scan [8] to detect statistically significant circular clusters of anomalous activity. They used properties of the pipe network to create a distance metric based on travel time between sensing nodes in order to define their "circles". However, they were not able to enforce connectivity constraints and take advantage of the topology of the network. Through our Additive GraphScan algorithm, we are able to search over connected subsets of the pipe network to find anomalous connected subgraphs. Berry et al. [2] have also considered the detection power of a network of imperfect sensors, showing that it is worth deploying a sensor network even when individual sensors have low detection probability. However, their experiments did not allow for sensors with false positives, making the detection and source tracing problems much easier to solve as compared to the more difficult scenario considered here.

Spatial scan statistics attempt to identify regions of interest or "hot spots". This is achieved by 
maximizing a scoring function $F(S)$, typically defined as the likelihood ratio $F(S)=\frac{\operatorname{Pr}\left(\operatorname{Data} \mid H_{1}(S)\right)}{\operatorname{Pr}\left(\operatorname{Data} \mid H_{0}\right)}$, over spatial regions $S$. In this expression $H_{1}(S)$ assumes increased activity in region $S$, and $H_{0}$ assumes regular behavior. This work monitors binary sensors $s_{i}$ each producing $c_{i} \sim$ Bernoulli (FPR) "triggers" under $H_{0}$ or $c_{i} \sim$ Bernoulli (TPR) "triggers" for $H_{1}(S)$ containing node $s_{i}$. This makes the expectation-based binomial scan statistic [8] a logical choice.

Spatial-temporal scan statistics incorporate the time dimension. It is standard to aggregate this temporal information over a time window $w$ so that $c_{i}=\sum_{t=1 \ldots w} c_{i}^{t}$. Once the temporal information has been aggregated for each window $w=1 \ldots W$, maximizing the spatial-temporal scan statistic for that window proceeds identically to the regular spatial scan statistic; it then maximizes over all window sizes from 1 to $W$. However, an inherent assumption in this aggregation of temporal information is that the affected spatial-temporal subset does not change over time. Therefore, this approach will be referred to as the Static scan method throughout this text.

The fundamental goal of this work is to relax this strong assumption on the spatial-temporal structure in order to increase the power to detect dynamic patterns that change the affected region over time. One simple approach is to optimize each of the $w$ time steps independently. This allows for each time step $t$ to identify an entirely different spatial region, but does not allow the sharing of information between time steps, possibly reducing detection power. This approach is referred to as the Independent scan method throughout this text.

As a compromise between Static and Independent methods, the Dynamic Subset Scan is proposed which enforces temporal consistency constraints to allow temporally adjacent time steps to share information forward and backward in time. As demonstrated below, this flexibility increases power to detect and track dynamic patterns while scaling to the size of real-world networks.

The rest of the paper is laid out as follows. Section II introduces temporal consistency constraints, applied to the expectation-based binomial scoring function, and demonstrates how the penalized scan statistic can be efficiently optimized over (not necessarily connected) dynamic subsets. Section III explains the Additive GraphScan algorithm, which efficiently identifies highscoring, connected subsets of data with an underlying graph structure. Section IV provides empirical results of the simulations of the water distribution network from the BWSN, comparing the Dynamic Subset Scan approach to several other approaches, and demonstrating improvements in detection, tracking, and source-tracing performance. Finally, Section V concludes the paper.

\section{Temporal Consistency Constraints}

This section has four sequential objectives. The first is to demonstrate how the expectationbased binomial (EBB) scoring function may incorporate additional constraints while remaining straightforward to optimize over all possible subsets $S$ (i.e., show that EBB can be written as an additive function over the data elements $s_{i} \in S$ ). Second, is to show that these constraints may be interpreted as the prior log-odds for a given node $s_{i}$ to be in the detected subset. The section continues by providing the formal definition of temporal consistency constraints based on a probabilistic generative model that incorporates both forward and backward temporal consistency. The section concludes with a description of the iterative optimization process that "lines up" the spatial-temporal region according to the provided temporal consistency constraints. 


\subsection{Additive Scoring Function and Additional Terms}

Conditioned on the false and true positive rates (FPR, TPR) of the sensors, the EBB statistic can be written as an additive set function over the data elements $s_{i} \in S$. This is an important feature for two reasons. First, additive functions are easy to optimize over all possible subsets. Without connectivity constraints, the optimization process is simply including all records making a positive contribution and excluding the rest. Determining the "most positive" connected subset is more complicated, and is covered in the Additive GraphScan section below. Second, additive functions allow for additional penalty terms $\Delta_{i}$ to be included at the element level while the total penalized scoring function remains additive and thus amenable to efficient optimization.

Theorem 1. The expectation-based binomial statistic may be written as $F(S)=\sum_{s_{i} \in S} \lambda_{i}$, where $\lambda_{i}$ depends only on the binary sensor response $c_{i}$ for sensor $s_{i}$ (i.e., whether that sensor triggers or not) as well as the false and true positive rates of the sensors in general.

Proof. The log-likelihood ratio form of the EBB scan statistic can be written as follows:

$$
\begin{aligned}
F(S) & =\log \frac{\operatorname{Pr}\left(\text { Data } \mid H_{1}(S)\right)}{\operatorname{Pr}\left(\text { Data } \mid H_{0}\right)} \\
& =\log \frac{\prod_{s_{i} \in S} \operatorname{Pr}\left(c_{i} \sim \text { Bernoulli }(\mathrm{TPR})\right)}{\left.\prod_{s_{i} \in S} \operatorname{Pr}\left(c_{i} \sim \text { Bernoulli(FPR }\right)\right)} \\
& =\log \prod_{s_{i} \in S} \frac{(\mathrm{TPR})^{c_{i}}(1-\mathrm{TPR})^{1-c_{i}}}{(\mathrm{FPR})^{c_{i}}(1-\mathrm{FPR})^{1-c_{i}}} \\
& =\sum_{s_{i} \in S}\left[c_{i} \log \left(\frac{\mathrm{TPR}}{\mathrm{FPR}}\right)+\left(1-c_{i}\right) \log \left(\frac{1-\mathrm{TPR}}{1-\mathrm{FPR}}\right)\right]
\end{aligned}
$$

Then $\lambda_{i}=c_{i} \log \left(\frac{\mathrm{TPR}}{\mathrm{FPR}}\right)+\left(1-c_{i}\right) \log \left(\frac{1-\mathrm{TPR}}{1-\mathrm{FPR}}\right)$.

Next, assume a bonus or penalty $\Delta_{i}$ for each $s_{i} \in S$. These can easily be incorporated into the score function. Define:

$$
F_{\text {pen }}(S)=F(S)+\sum_{s_{i} \in S} \Delta_{i}=\sum_{s_{i} \in S}\left(\lambda_{i}+\Delta_{i}\right)
$$

$F_{p e n}(S)$ is a penalized form of the EBB scan statistic that is still additive over the data elements $s_{i}$. Note that the $\Delta_{i}$ terms are assumed to be a function of only the given data element $s_{i}$; they cannot depend on the entire subset $S$. This as a limitation of the current work and will be investigated in extensions to more sophisticated penalties in future work.

\subsection{Prior Log-odds Interpretation}

These soft constraints, $\Delta_{i}$, have a convenient interpretation as the prior log-odds that each data element $s_{i}$ will be included in the detected subset. Let $p_{i}$ be the prior probability that data element $s_{i}$ will be contained in the detected subset. Then $\Delta_{i}$ can be defined as $\log \left(\frac{p_{i}}{1-p_{i}}\right)$. The prior $\log$ probability of selecting a subset $S$ is then: 


$$
\begin{aligned}
\log \operatorname{Pr}(S) & =\log \left(\prod_{s_{i} \in S} p_{i} \prod_{s_{i} \notin S}\left(1-p_{i}\right)\right) \\
& =\sum_{s_{i} \in S}\left(\log p_{i}-\log \left(1-p_{i}\right)\right)+\sum_{i=1}^{N} \log \left(1-p_{i}\right) \\
& =\sum_{s_{i} \in S} \Delta_{i}-\sum_{i=1}^{N} \log \left(1+\exp \left(\Delta_{i}\right)\right) .
\end{aligned}
$$

However, note that the term $\sum_{i=1}^{N} \log \left(1+\exp \left(\Delta_{i}\right)\right)$ is constant and does not affect the probability of selecting any particular subset. Thus, this term can be ignored when optimizing over all subsets of the data, and be subtracted once the highest-scoring subset of the data has been identified. When $\Delta_{i}>0$, record $s_{i}$ is more likely to be included in the detected subset, and the opposite is true when $\Delta_{i}<0$. When $\Delta_{i}=0$ for all $i$, which is the default setting for spatial-temporal scan statistics, then every subset $S$ is considered equally likely a priori.

\subsection{Derivation of $\Delta_{i}^{t}$}

This section derives the formulas for $\Delta_{i}^{t}$ that correspond to the following generative model for temporal consistency. Let $p_{i}^{t}$ be the prior probability that data element $s_{i}$ will be contained in the detected subset $S^{t}$ on time step $t$. Let $x_{i}^{t}$ be 1 if data element $s_{i}$ is included in $S^{t}$, and 0 otherwise. Let $n_{i}^{t}$ be the number of neighbors of $s_{i}$ that are included in $S^{t}$, and let $k_{i}$ be the degree of node $s_{i}$. Then the generative model of event propagation, which incorporates temporal consistency constraints, is defined as:

$$
\log \left(\frac{p_{i}^{t}}{1-p_{i}^{t}}\right)=\beta_{0}+\beta_{1} x_{i}^{t-1}+\beta_{2} \frac{n_{i}^{t-1}}{k_{i}} .
$$

As a concrete example of the interpretation of this model, assume $\beta_{0}=-1.5, \beta_{1}=5$, and $\beta_{2}=0$. Then, if a node is included in the previous detected subset, $S^{t-1}$, it has a $97 \%$ prior probability of being included in the current detected subset, $S^{t}$. If it was not included in the previous subset, then it only has an $18 \%$ probability of being included in the current subset. When $\beta_{2}>0$, the proportion of neighbors $j$ included in $S^{t-1}$ will further influence the prior probability of $s_{i}$ being included in the current subset.

Consider $\Delta_{i}^{t}$ as the total impact of including $x_{i}^{t}$ on the overall penalized log-likelihood ratio score $F(S)$, as compared to the score $F\left(S \backslash x_{i}^{t}\right)$ when $x_{i}^{t}$ is excluded. The log-linear model of $p_{i}^{t}$ above provides:

$$
\begin{aligned}
\Delta_{i}^{t}=\left(\log \left(p_{i}^{t}\right)-\log \left(1-p_{i}^{t}\right)\right) & +\sum_{j \in S^{t+1}}\left(\log \left(p_{j}^{t+1} \mid x_{i}^{t}\right)-\log \left(p_{j}^{t+1} \mid \bar{x}_{i}^{t}\right)\right) \\
& +\sum_{j \notin S^{t+1}}\left(\log \left(1-p_{j}^{t+1} \mid x_{i}^{t}\right)-\log \left(1-p_{j}^{t+1} \mid \bar{x}_{i}^{t}\right)\right) .
\end{aligned}
$$

In equation (2), the initial difference results from the prior probability of $x_{i}^{t}$, conditioned on $x_{i}^{t-1}$ and its number of included neighbors $n_{i}^{t-1}$ from the previous time step. This difference can 
be calculated directly from the model:

$$
\log \left(p_{i}^{t}\right)-\log \left(1-p_{i}^{t}\right)=\beta_{0}+\beta_{1} x_{i}^{t-1}+\beta_{2} \frac{n_{i}^{t-1}}{k_{i}} .
$$

The two sums in (2) account for the fact that including $x_{i}^{t}$ changes the prior probabilities of $x_{i}^{t+1}$ and its neighbors $n_{i}^{t+1}$ for the next time step. These sums can be rewritten as:

$$
\begin{aligned}
& \sum_{j \in S^{t+1}}\left(\log \left(p_{j}^{t+1} \mid x_{i}^{t}\right)-\log \left(p_{j}^{t+1} \mid \bar{x}_{i}^{t}\right)\right)+\sum_{j \notin S^{t+1}}\left(\log \left(1-p_{j}^{t+1} \mid x_{i}^{t}\right)-\log \left(1-p_{j}^{t+1} \mid \bar{x}_{i}^{t}\right)\right) \\
= & \sum_{j \in S^{t+1}}\left(\beta_{0}+\beta_{1} x_{j}^{t}+\beta_{2} \frac{n_{j}^{t}}{k_{j}} \mid x_{i}^{t}\right)-\sum_{j} f\left(\beta_{0}+\beta_{1} x_{j}^{t}+\beta_{2} \frac{n_{j}^{t}}{k_{j}} \mid x_{i}^{t}\right) \\
- & \sum_{j \in S^{t+1}}\left(\beta_{0}+\beta_{1} x_{j}^{t}+\beta_{2} \frac{n_{j}^{t}}{k_{j}} \mid \bar{x}_{i}^{t}\right)+\sum_{j} f\left(\beta_{0}+\beta_{1} x_{j}^{t}+\beta_{2} \frac{n_{j}^{t}}{k_{j}} \mid \bar{x}_{i}^{t}\right),
\end{aligned}
$$

where the function $f(x)=\log (1+\exp (x))$. Next, note that the contributions to equation (4) are equal to 0 for all nodes $j$ except for node $i$ and its neighbors. For $j=i$, the corresponding terms in (4) simplify to:

$$
\beta_{1} x_{i}^{t+1}+f\left(\beta_{0}+\beta_{2} \frac{n_{i}^{t}}{k_{i}}\right)-f\left(\beta_{0}+\beta_{1}+\beta_{2} \frac{n_{i}^{t}}{k_{i}}\right) .
$$

For each neighbor $j$ of $i$, the corresponding terms in (4) simplify to:

$$
\beta_{2}\left(\frac{x_{j}^{t+1}}{k_{j}}\right)+f\left(\beta_{0}+\beta_{1} x_{j}^{t}+\beta_{2} \frac{n_{j}^{t}}{k_{j}}\right)-f\left(\beta_{0}+\beta_{1} x_{j}^{t}+\beta_{2} \frac{n_{j}^{t}+1}{k_{j}}\right) .
$$

Adding the contributions of equations (3), (5), and (6) provides:

$$
\begin{aligned}
\Delta_{i}^{t} & =\beta_{0}+\beta_{1}\left(x_{i}^{t-1}+x_{i}^{t+1}\right)+\beta_{2}\left(\frac{n_{i}^{t-1}}{k_{i}}+\sum_{j \in S^{t+1}} \frac{1}{k_{j}}\right) \\
& +f\left(\beta_{0}+\beta_{2} \frac{n_{i}^{t}}{k_{i}}\right)-f\left(\beta_{0}+\beta_{1}+\beta_{2} \frac{n_{i}^{t}}{k_{i}}\right) \\
& +\sum_{j} f\left(\beta_{0}+\beta_{1} x_{j}^{t}+\beta_{2} \frac{n_{j}^{t}}{k_{j}}\right)-\sum_{j} f\left(\beta_{0}+\beta_{1} x_{j}^{t}+\beta_{2} \frac{n_{j}^{t}+1}{k_{j}}\right),
\end{aligned}
$$

where the sums are taken over all neighbors $j$ of $i$. In the special case of $\beta_{2}=0$, equation (7) simplifies to:

$$
\Delta_{i}^{t}=\beta_{0}+\beta_{1}\left(x_{i}^{t-1}+x_{i}^{t+1}\right)+f\left(\beta_{0}\right)-f\left(\beta_{0}+\beta_{1}\right) .
$$

Note that, when $\beta_{2}=0, \Delta_{i}^{t}$ can be computed exactly. When $\beta_{2} \neq 0, \Delta_{i}^{t}$ can must be approximated assuming that $\beta_{0}+\beta_{2} \ll 0$ and $\beta_{0}+\beta_{1} \gg 0$. Noting that $f(x) \approx 0$ when $x \ll 0$, and 
$f(x) \approx x$ when $x \gg 0$, provides:

$$
\begin{aligned}
\Delta_{i}^{t} \approx & \beta_{0}+\beta_{1}\left(x_{i}^{t-1}+x_{i}^{t+1}\right)+\beta_{2}\left(\frac{n_{i}^{t-1}}{k_{i}}+\sum_{j \in S^{t+1}} \frac{1}{k_{j}}\right)-\left(\beta_{0}+\beta_{1}+\beta_{2} \frac{n_{i}^{t}}{k_{i}}\right)+ \\
& \sum_{j \in S^{t}}\left(\beta_{0}+\beta_{1}+\beta_{2} \frac{n_{j}^{t}}{k_{j}}\right)-\sum_{j \in S^{t}}\left(\beta_{0}+\beta_{1}+\beta_{2} \frac{n_{j}^{t}+1}{k_{j}}\right) \\
= & \beta_{1}\left(x_{i}^{t-1}+x_{i}^{t+1}-1\right)+\beta_{2}\left(\frac{n_{i}^{t-1}}{k_{i}}+\sum_{j \in S^{t+1}} \frac{1}{k_{j}}-\sum_{j \in S^{t}}\left(\frac{1}{k_{i}}+\frac{1}{k_{j}}\right)\right) .
\end{aligned}
$$

However, equation (9) assumes knowledge of which other elements are contained in $S^{t}$, and this information would not be known in advance. Thus the final sum over $j \in S^{t}$ is approximated with half the corresponding sum over all neighbors $j$ of $i$ :

$$
\Delta_{i}^{t} \approx \beta_{1}\left(x_{i}^{t-1}+x_{i}^{t+1}-1\right)+\beta_{2}\left(\frac{n_{i}^{t-1}}{k_{i}}+\sum_{j \in S^{t+1}} \frac{1}{k_{j}}-\frac{1}{2} \sum_{j}\left(\frac{1}{k_{i}}+\frac{1}{k_{j}}\right)\right) .
$$

The intuitive role of $\Delta_{i}^{t}$ is that it must simultaneously make $S^{t}$ appear likely to have been generated from $S^{t-1}$ and able to generate $S^{t+1}$, thus conveying temporal consistency information both forwards and backwards in time.

\subsection{Iterative Convergence}

The previous section provided definitions and interpretations for $F_{p e n}(S)=\sum_{s_{i}^{t} \in S}\left(\lambda_{i}^{t}+\Delta_{i}^{t}\right)$ for the EBB scan statistic with temporal consistency constraints. However, recall that the values of $\Delta_{i}^{t}$ for a given time step $t$ depend on the detected subsets at $t-1$ and $t+1$, which creates a computational paradox. To solve this, the Dynamic Subset Scan uses an iterative method that converges to a (local) optimum. To better approach the global optimum, multiple restarts and simulated annealing (which gradually increases the strength of the $\Delta_{i}^{t}$ from 0 to their full values) are wrapped around steps (3)-(13) in the algorithm below.

\section{Additive GraphScan}

The previous section outlined how the expectation-based binomial (EBB) scoring function may be penalized with temporal consistency constraints $F_{p e n}(S)=\sum_{s_{i} \in S}\left(\lambda_{i}+\Delta_{i}\right)$ while remaining an additive set function over the data elements $s_{i} \in S$. Optimizing additive functions without connectivity constraints is very straightforward and consists of including all records with positive contributions $\left(\lambda_{i}+\Delta_{i}>0\right)$ and excluding the rest. Enforcing hard connectivity constraints on additive functions (i.e. determining the "most positive" connected subset) is an interesting and difficult problem. For example, not all nodes making positive contributions will be included in a high-scoring connected subset because they are likely disconnected in the underlying graph structure. Also, a high-scoring connected subset may include a node with a negative contribution in order to connect two positive nodes.

The GraphScan algorithm [13] exactly identifies the highest scoring connected subset for any scoring function that satisfies the Linear-Time Subset Scanning (LTSS) property [11]. It is trivially 


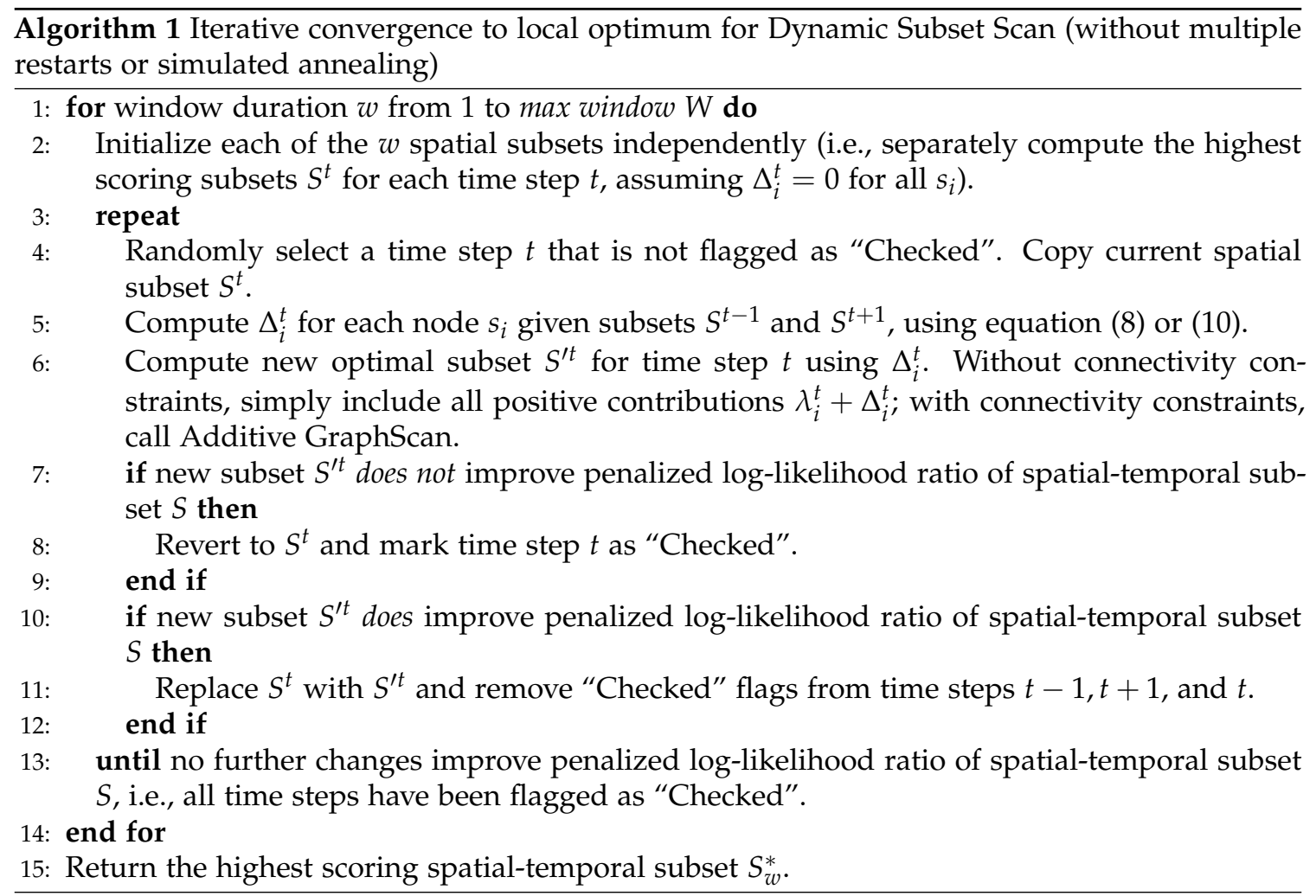

shown that additive functions satisfy LTSS, and therefore GraphScan could be used to determine the highest scoring ("most positive", in the case of an additive scoring function) connected subset. However, GraphScan is designed to optimize over more complex scoring functions; most importantly, its computation time is exponential in the graph size and therefore it does not scale well in this setting. Therefore, Additive GraphScan is proposed as an efficient heuristic alternative to GraphScan which can be used to identify high-scoring (most positive) connected subsets in a given graph structure with real-valued weights at each node.

\subsection{Additive GraphScan Algorithm}

Additive GraphScan makes use of the following notation. $w(n)$ is the real-valued weight of node $n$. A path $p$ is any connected subgraph of nodes. $w(p)$ is the sum of weights for every node in the path. $g(p)$ is the gain that would result from merging path $p$ into a single node. It is the difference between the weight of the resulting merged node and the highest weighted node in the path. Identifying and merging paths with positive gains is an integral part of Additive GraphScan. $g\left(n, p^{*}\right)$ is the gain that would result from merging two paths together. The first path, $p^{*}$, is a previously identified path of interest with positive gain. The second path is the shortest path between node $n$ and any point along path $p^{*} . g\left(n, p^{*}\right)$ is the difference between the weight of the resulting merged paths and $\max \left(w(n), w\left(p^{*}\right)\right)$.

$p w(n)$ is the pathweight of a node used when calculating single source, shortest paths traversing through the node. Note the difference between the weight of a node $w(n)$ (which may be positive or negative and is used in the gain calculations above) and the pathweight of a node $p w(n)$ (which is non-negative and used in shortest path calculations). Pathweights of positive 
nodes are set to 0 , reflecting no penalty (or reward) for traversing positive nodes while identifying shortest paths. Pathweights for negative nodes with no positive neighbors are $-w(n)$. Pathweights for negative nodes with positive neighbors have

$$
p w(n)=-\min \left(0, w(n)+\sum_{\text {pos neighbors, } n_{i}} \frac{w\left(n_{i}\right)}{\operatorname{degree}\left(n_{i}\right)}\right) .
$$

These positive weights may be thought as uniformly "diffusing" over their negative neighbors and then using this altered weight as the pathweight for negative nodes with positive neighbors. In the case where a large positive node overwhelms its negative neighbor, the negative neighbor's pathweight is set to 0 .

Finally, $s\left(n_{a}, n_{b}, n_{c}\right)$ determines a fourth node, $n_{s}$ in the graph as a Steiner point for $n_{a}, n_{b}$, and $n_{c}$. A Steiner point in this setting is a node that forms the shortest interconnect between the three provided nodes using the pathweights of the graph. $s\left(n_{a}, n_{b}, n_{c}\right)$ returns the shortest interconnecting path formed between the three nodes going through $n_{s}$.

Some basic pre-processing may be applied to the graph before running Additive GraphScan. For example, any positive node with a positive neighbor may be merged together into a larger, single positive node (adding their weights) and repeated until no further merges exist. Also, any negative nodes with degree of 1 or less may be recursively removed because these are guaranteed to not be included in a high scoring connected subset. Lastly, any negative node with at least two positive neighbors may be merged into a single node if the resulting merged node has a higher weight than any individual positive neighbor. Additive GraphScan can then be applied to the pre-processed graph. The Additive GraphScan algorithm scales as $O\left(k N^{2}\right)=O\left(N^{2.5}\right)$, dominated by steps (3) and (5).

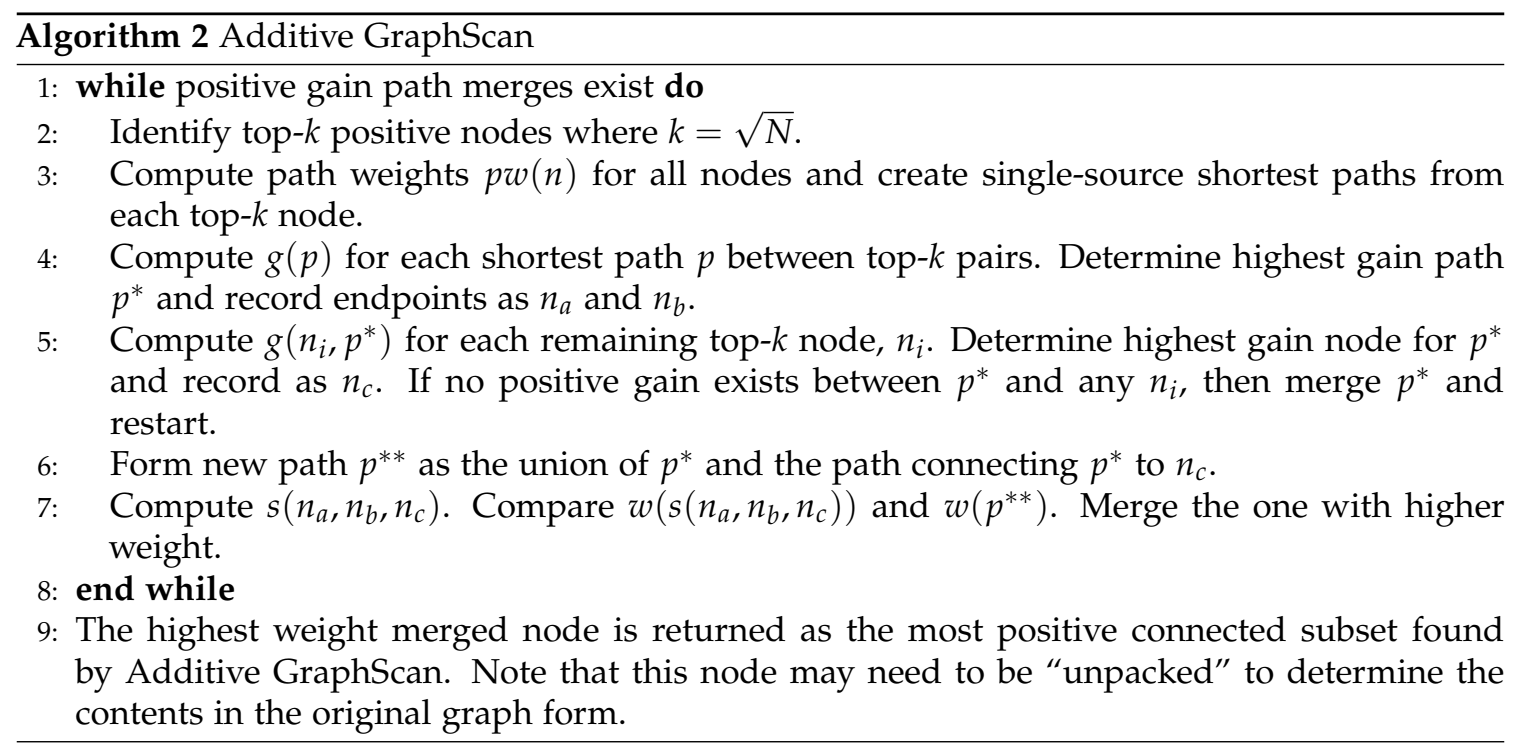




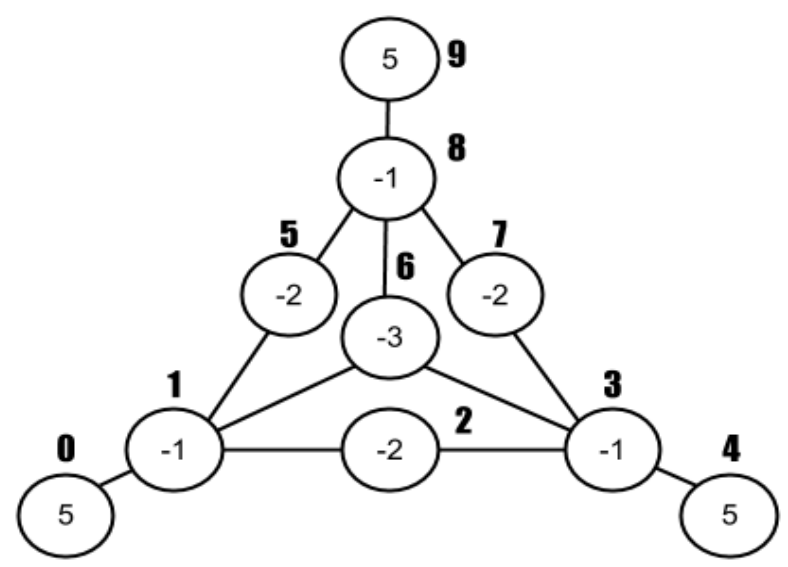

Figure 1: An example graph to demonstrate the Additive GraphScan algorithm. The large bolded numbers are node identifiers and the small numbers within each node are the nodes' corresponding weights. The most positive subgraph consists of nodes $\{0,1,6,3,4,8,9\}$ and is correctly identified by Additive GraphScan.

\subsection{Additive GraphScan Example}

This section concludes by applying Additive GraphScan to a sample pre-processed graph found in Figure 1. The most positive connected subgraph consists of nodes $\{0,1,6,3,4,8,9\}$ where node 6 is the Steiner point used to connect nodes 0, 4, and 9. Additive GraphScan correctly identifies this subgraph even though node 6 is not on the shortest paths connecting nodes 0 and 4 or nodes 4 and 9. A key insight into the strong performance of Additive GraphScan is delaying path merges while searching for a potential Steiner point. Begin at step (2:) Nodes 0, 4, and 9 are identified as the top- $k$ nodes. (3:) Dijkstra's algorithm is called on nodes 0,4 , and 9 providing single-source shortest path information from each of them. (4:) The shortest path from node 0 to node $4, p^{*}=\{0,1,2,3,4\}$, has highest gain of $g\left(p^{*}\right)=(5-1-2-1+5)-5=+1$. Because a positive gain path was found between nodes $n_{a}=0$ and $n_{b}=4$, Additive GraphScan continues searching for a third node, $n_{c}$. (5,6:) Node $n_{c}=9$ is found with $p^{* *}=\{0,1,2,3,4,7,8,9\}$ and $w\left(p^{* *}\right)=8$. (7:) Calculate a Steiner point for nodes 0, 4, and 9 and note that node 6 forms the shortest interconnect between these three points. This interconnect is formed by the nodes $\{0,1,6,3,4,8,9\}$ and $w(s(0,4,9))=5-1-3-1+5-1+5=9$. Because $w(s(0,4,9))>w\left(p^{* *}\right)$ the Steiner interconnect $s(0,4,9)$ is condensed into a single node with weight 9 . After this merge, no more positive gain path merges exist and the loop exits. (9:) The highest scoring connected subset is then $\{0,1,6,3,4,8,9\}$. Notice that greedily merging either $p^{*}$ or $p^{* *}$ would have resulted in a sub-optimal merge.

\section{Results}

\subsection{Comparison of Additive GraphScan vs. GraphScan}

This section compares the fast heuristic, Additive GraphScan, to the slower, but exact, GraphScan algorithm. First, a runtime analysis is presented comparing the two optimization algorithms. The much larger "network 2" provided in the Battle of the Water Sensor Networks [1] is used to create connected subgraphs of various sizes from 50 to 500 nodes from the network. The graphs are processed with three different scans: Dynamic Subset Scan with GraphScan, Dynamic Subset 


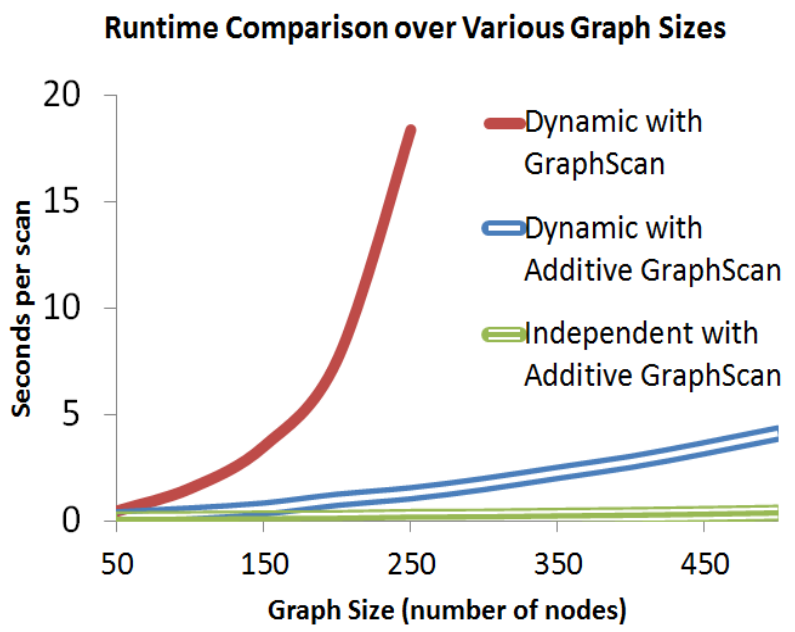

Figure 2: Runtime comparisons for the Dynamic Subset Scan with GraphScan and Additive GraphScan as the optimization algorithm. Independent Scan with Additive GraphScan is also shown.

Scan with Additive GraphScan, and Independent with Additive GraphScan. The average runtime for each method is reported and are shown in Figure 2.

GraphScan begins to struggle with graph sizes of 250 nodes while Additive GraphScan quickly scans graphs of 500 nodes in approximately 4.1 seconds. Independent with Additive GraphScan processed the entire 12,000+ node "Network 2" in 221 seconds while Dynamic with Additive GraphScan required 1830 seconds (approximately a half hour). This difference represents the additional calls to Additive GraphScan required by Dynamic Subset Scan to "align" the individual spatial subsets according to the temporal consistency constraints.

The comparison of Additive GraphScan and GraphScan is concluded by analyzing the scores of the spatial-temporal subsets identified by the scanning methods using both Additive GraphScan and GraphScan. The approximation ratio results compare the highest-scoring subsets found by Additive GraphScan and GraphScan as a percentage averaged over 2000 simulations. Table 1 provides detailed information for the approximation ratios. The ratios over $100 \%$ in the Dynamic cases reflect the noise in the iterative convergence process outlined above in Section 2.4. To be clear, Additive GraphScan is not identifying a higher scoring subgraph than GraphScan for an individual time slice. However, the local optimum after the iterative convergence of Additive GraphScan-based optimizations at each step may have a higher score than the local optimum reached with GraphScan-based optimizations at each time step. The Static and Independent methods do not use this iterative convergence process to identify the highest scoring spatialtemporal region and may reflect a more direct comparison between the performance of Additive GraphScan and GraphScan. The ratio does not fall below 98.4\% indicating that Additive Graphscan is providing a huge speed increase with minimal loss of accuracy compared to scan statistics using GraphScan.

\subsection{Detecting, Tracking, and Source-Tracing Plumes}

This section evaluates the detection, tracking, and source-tracing abilities of the Dynamic Subset Scan. The 129-node "Network 1" from the Battle of the Water Sensor Networks [1] served as the test bed for these evaluations. Two simulations were performed. One with sensors at FPR $=0.1$ and $\mathrm{TPR}=0.9$ and a second with weaker sensors at $\mathrm{FPR}=0.2$ and $\mathrm{TPR}=0.8$. All results below 
Table 1: Approximation ratios comparing scores for Additive GraphScan and GraphScan for multiple methods and FPR and TPR.

\begin{tabular}{|l|cc|cc|}
\hline method & Background(0.1) & Injects(0.9) & Background(0.2) & Injects(0.8) \\
\hline Static & $100.00 \%$ & $100.00 \%$ & $99.15 \%$ & $99.83 \%$ \\
Independent & $100.00 \%$ & $99.99 \%$ & $98.48 \%$ & $99.65 \%$ \\
Dynamic & $100.59 \%$ & $101.44 \%$ & $99.70 \%$ & $100.50 \%$ \\
\hline
\end{tabular}

Table 2: Summary of learned parameter values

\begin{tabular}{|l|c|c|c|c|c|}
\hline method & FPR & TPR & $\beta_{0}$ & $\beta_{1}$ & $\beta_{2}$ \\
\hline Dynamic & 0.1 & 0.9 & -1.3 & 6.6 & 1.8 \\
Dynamic & 0.2 & 0.8 & -1.3 & 3.6 & 2.0 \\
Dynamic Alt. & 0.1 & 0.9 & -1.1 & 8.2 & 0 \\
Dynamic Alt. & 0.2 & 0.8 & -1.2 & 2.4 & 0 \\
\hline
\end{tabular}

are averaged over 200 contaminant plumes simulated for 12 hours each. A separate 100-plume training set was used for cross-validation for the scan statistics that required learning parameters.

Comparisons are made for 4 different spatial-temporal scan statistics:

- Static scan does not allow the detected spatial region to change over time.

- Independent scan allows the detected spatial region to change over time but does not share temporal information between time steps.

- Dynamic scan allows the detected spatial region to change over time and uses temporal consistency constraints to "align" the individual time steps.

- Dynamic Alt. scan is similar to Dynamic scan but does not use any information from neighbors when enforcing temporal consistency constraints, i.e., forces $\beta_{2}=0$ when learning the model parameters.

For the temporal component of the scans, max window size $W=12$ is used. This allows Static, Dynamic, and Dynamic Alt. to detect a spatial-temporal subset between 1 and 12 hours in duration. However, for the Independent scan, the highest scoring spatial-temporal region will always be maximum duration. This consequence of the Independent scan is discussed further below.

The $\beta_{0} \ldots \beta_{2}$ parameters for the Dynamic scan, and the $\beta_{0}$ and $\beta_{1}$ parameters for Dynamic Alt. were set using a grid search on the separate 100-plume training set. The parameter values that maximized spatial-temporal overlap in the training data are shown in Table 2. Note the large changes in $\beta_{1}$ when moving from the easier to harder scenarios, while $\beta_{0}$ and $\beta_{2}$ remain relatively constant.

Figure 3 reports the average time required by each method to detect a contaminant plume for various false positive rates. These results were calculated by processing 2160 "background" hours (approximately 3 months of data) with no contaminants. These were compared with scores produced by the scan statistics during the 200 simulated plumes. The 0 false positive alarms 


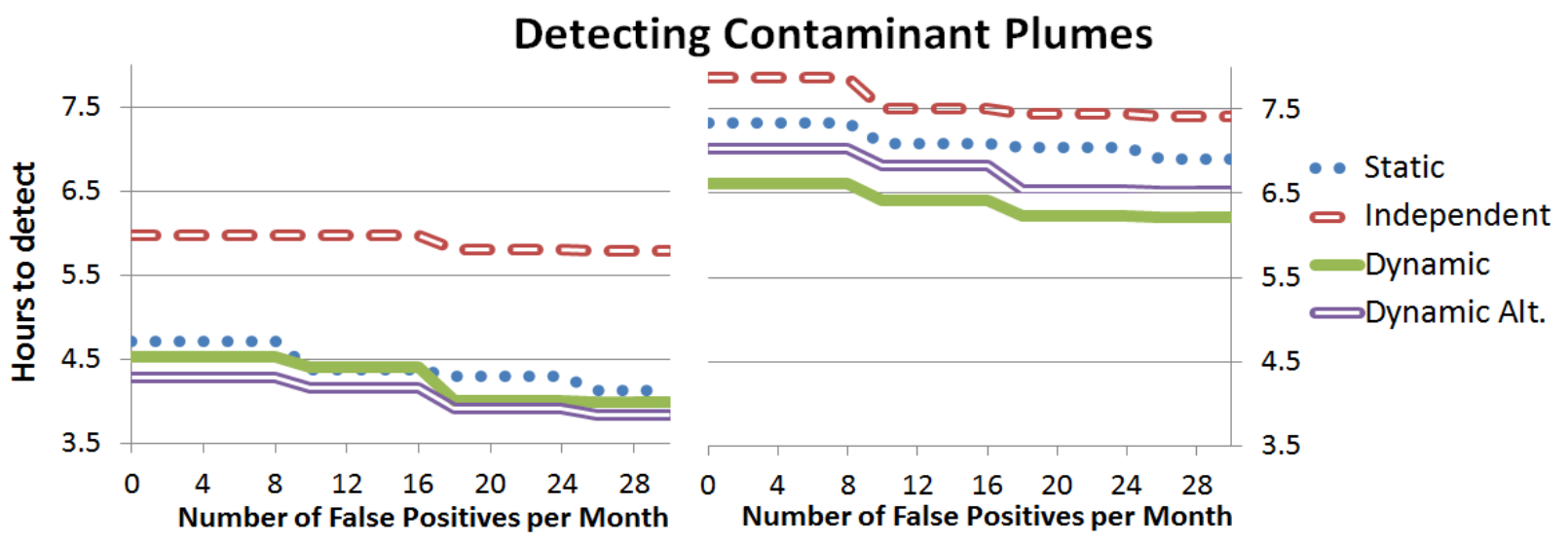

Figure 3: Detection results for $F P R=0.1$ and $T P R=0.9$ sensors on the left and $F P R=0.2$ and $T P R=0.8$ sensors on the right. The detection results are reported through Activity Monitoring Operating Characteristic (AMOC) curves. These show the average time required for each method to detect a contaminant in the system, assuming a fixed number of allowable false positives per month.

interpretation is that it took 4.3 hours (on average) for the scores produced by Dynamic Scan to exceed the largest score found by Dynamic Scan in the 2160 "background" hours. As the threshold for detection is lowered, the number of false positive alarms increases but the time to detect decreases, as shown by the Activity Monitoring Operating Characteristic (AMOC) curves [4] in Figure 3.

The Static, Dynamic, and Dynamic Alt. methods achieve similar power for event detection in the easier scenario (FPR $=0.1, \mathrm{TPR}=0.9$ ). However, Dynamic achieves the overall best performance (6.62 hours to detect at 0 false positives) when detecting a weaker signal (FPR $=0.2$, TPR $=0.8$ ). Note the influence that a node's neighbors have on distinguishing performance between Dynamic and Dynamic Alt. in the 0.2/0.8 scenario. The easier scenario did not require additional information from neighbors in order to obtain similar performance, but this information is important when working with weaker sensors. The Independent method's poor performance is due to the relatively high subset scores found by Independent when no contaminant is present. Its unconstrained flexibility allows it to overfit to noise in the background, making detection of a true contamination event more difficult.

Figure 5 reports the methods' tracking ability over the duration of a spreading contaminant plume (12 hours). A scan statistic's tracking ability is measured through spatial-temporal overlap. Spatial-temporal overlap is a combination of precision and recall applied to spatial-temporal subsets. A measure of 1.0 corresponds to perfect agreement between the affected and detected spatial-temporal regions, while 0.0 means the affected and detected regions are disjoint. For two spatial-temporal subsets, Affected and Detected, the overlap is defined as: $\frac{\mid \text { Affected } \cap \text { Detected } \mid}{\mid \text { Affected } \cup \text { Detected } \mid}$. See Figure 4 for details.

The relative performance of the Static and Dynamic methods in the easier scenario demonstrates Static's lack of tracking ability as the plume grows over time. Static's tracking performance quickly levels off while Dynamic continues to achieve higher spatial-temporal overlap over the course of the contamination event. This increase in performance is due to the (constrained) flexibility allowed to the Dynamic Subset Scan. The difference in tracking performance between Static and Dynamic methods is not as large in the harder scenario, but note the importance of incorporating information from a node's neighbors. The poor performance of the Independent 


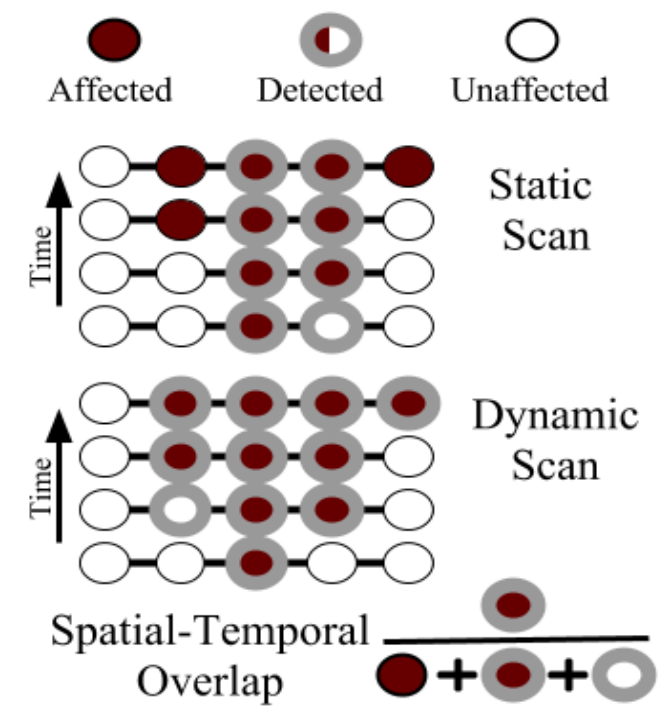

Figure 4: This figure demonstrates the calculation of spatial-temporal overlap for plume tracking. A plume spreads through a simple 5-node line graph over the course of four time steps. Affected nodes turn from white to red as the contaminant spreads. The Static scan method is constrained to keep the exact same detected spatial region throughout the event duration. Hence, it may fail to capture the plume at later time steps. Dynamic Scan allows the detected spatial region to change at each time step, tracking the plume as it spreads. Due to connectivity constraints, both methods must return a connected subgraph as the detected spatial region at each time step. Spatial-temporal overlap is penalized for both false positives and false negatives. A measure of 1.0 corresponds to perfect agreement between the affected and detected spatial-temporal regions, while 0.0 means that the affected and detected regions are disjoint.

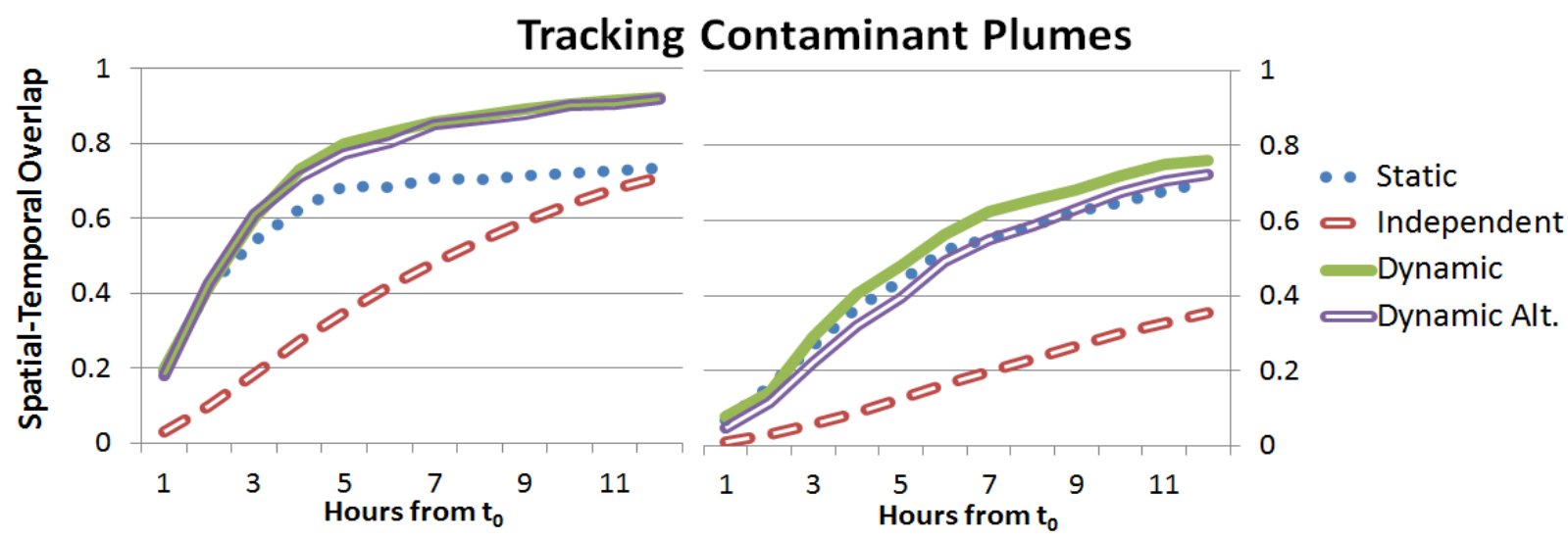

Figure 5: Tracking results for $F P R=0.1$ and $T P R=0.9$ sensors on the left and $F P R=0.2$ and $T P R=0.8$ sensors on the right. Tracking ability is measured by reporting the spatial-temporal overlap of the detected and affected subsets over the course of 12 hours. 


\section{Source-Tracing Contaminant Plumes}

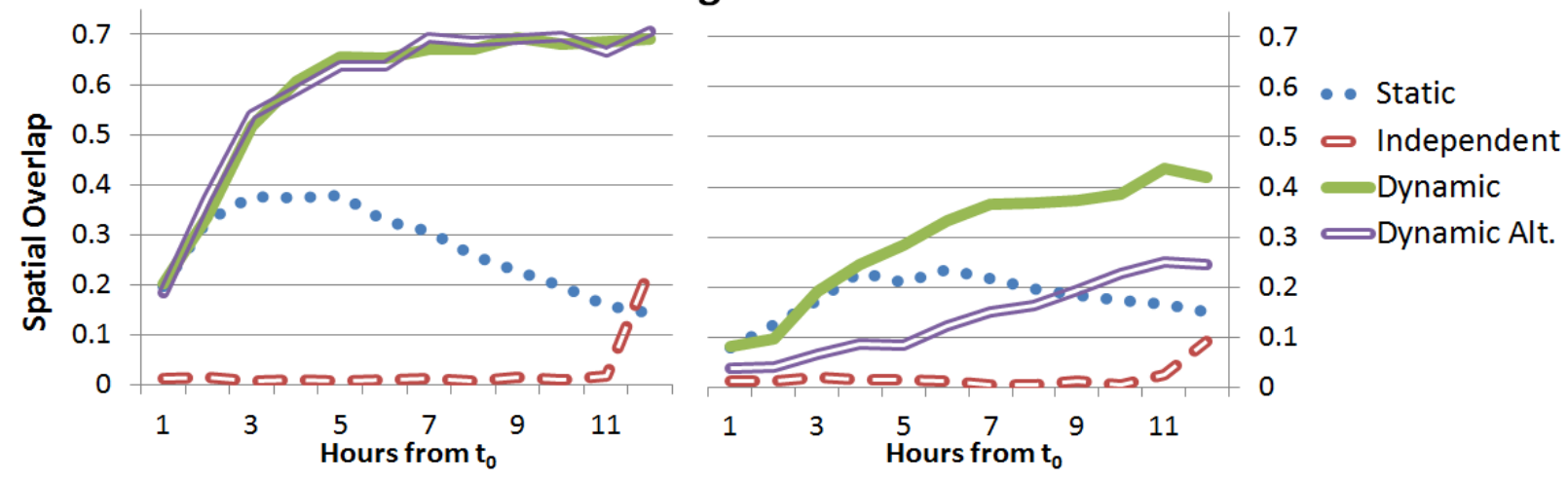

Figure 6: Source-tracing results for $F P R=0.1$ and $T P R=0.9$ sensors on the left and $F P R=0.2$ and $T P R=0.8$ sensors on the right. Source-tracing ability is measured by reporting the spatial overlap between the earliest detected spatial region and the original affected node(s).

scan, particularly in the early stages of the contamination event, is due to its tendency to report spatial-temporal regions of maximum duration which are not a good match to the true affected region.

Figure 6 reports the methods' ability to identify where the contaminant originated over the duration of the plume (12 hours). This is measured through purely spatial overlap between the earliest time step in the detected region and the source node(s) of the plume. Note that it is possible for a quickly spreading plume to affect multiple nodes within the first hour. In such cases, all of these nodes are treated as source nodes.

The source-tracing results clearly demonstrate the advantage of sharing information between time steps during the optimization process. Static's ability to identify the source nodes actually decreases over the course of the contamination event as more information is gathered. Exploring this result further, note that Static has very high spatial recall (0.995) but very low precision (0.144) for identifying the source nodes on hour 12. This suggests that Static tends to return very large subsets at the later stages of the plume. The large regions returned by the Static method harm its ability to accurately identify the source of the contaminant.

The key to the Dynamic Subset Scan's success for source-tracing is the backwards flow of temporal consistency information allowed in our model. Dynamic is able to change the detected subset for previous time steps based on new, more current data. This gives it superior sourcetracing abilities in both the easier $(0.1 / 0.9)$ and harder $(0.2 / 0.8)$ scenarios. The importance of including neighbor information is evident in the harder scenario: while the Dynamic method achieves similar performance to Static during the early stages of the contamination event and much better performance in the later stages, the performance of Dynamic Alt. (which does not use neighbor information) does not surpass Static until the ninth hour. Finally, note the substantial increase in performance of the Independent method at hour 12, though its overall performance is still low. This is an artifact of Independent preferring to return 12-hour regions.

\section{Conclusions}

This work introduced the Dynamic Subset Scan for detecting, tracking, and source-tracing $d y$ namic patterns that change the affected subset over time. This novel extension of the well-known 
spatial and subset scan statistics is composed of two main contributions. First is the incorporation of temporal consistency constraints that may be enforced on temporally adjacent, spatial subsets. These constraints are a fruitful compromise between traditional spatial-temporal scan statistics that do not allow the detected region to change over time (Static) and the other extreme where temporal information is ignored (Independent). The key insight to enforcing temporal consistency constraints is recognizing that the expectation-based binomial scoring function may be written as an additive function over the data records. This allows for additional terms (constraints) to be included in the penalized log likelihood ratio while remaining efficient to optimize. Critically, these temporal consistency constraints were derived to allow temporal information to be shared both forward and backward in time.

The second novel contribution is the Additive GraphScan algorithm, which allows the Dynamic Subset Scan to enforce both soft temporal consistency constraints and hard connectivity constraints while scaling to large, real world networks. Additive GraphScan is a fast, heuristic alternative to GraphScan. However, the results demonstrate an approximation ratio of over $99 \%$, suggesting a very small sacrifice for dramatic gains in speed and scalability.

The Dynamic Subset Scan was evaluated on data provided through the "Battle of the Water Sensor Networks" [1]. Dynamic scan succeeded in detecting contamination events sooner and tracking these events more accurately compared to other competing methods. The gains were due to Dynamic Scan's constrained flexibility: competing methods either failed to capture the dynamics of the spreading plume (Static) or were susceptible to over-fitting from lack of constraints (Independent). In scenarios with a weaker signal to be detected, incorporating information from a node's neighbors in the Dynamic Scan proved worthwhile, leading to substantial gains in performance on the detection, tracking, and source-tracing tasks.

In summary, relaxing constraints on spatial-temporal region shape must be done carefully. Strict temporal constraints work well when the affected subset of the data does not change over time. However, removing them completely in order to track dynamic patterns performs poorly as shown by the Independent method results. Dynamic Subset Scan with temporal consistency and connectivity constraints provides a scalable solution for future work in dynamic pattern detection in graph-based or sensor network data.

\section{Acknowledgment}

A special thanks to Professor Daniel Neill and the Event and Pattern Detection Laboratory for their (too many?) ideas and multiple edits of this work. Also, thanks to Yating Zhang who helped me think through Additive GraphScan at the implementation level. Thanks to R. Ravi for providing insights on the use of Steiner points. This work was partially supported by NSF grants IIS-0916345, IIS-0911032, and IIS-0953330.

\section{References}

[1] Avi Ostfeld et al. The battle of water sensor networks: A design challenge for engineers and algorithms. Journal of Water Resources Planning and Management, 134(6):556-568, 2008.

[2] J. Berry, R. D. Carr, W. Hart, V. J. Leung, C. A. Phillips, and J. P. Watson. Designing contamination warning systems for municipal water networks using imperfect sensors. Journal of Water Resources Planning and Management, 135(4):253-263, 2009. 
[3] J. Berry, L. Fleischer, W. Hart, and C. Phillips. Sensor placement in municipal water networks. J. Water, 131:237-243, 2003.

[4] T. Fawcett and F. Provost. Activity monitoring: noticing interesting changes in behavior. In Proc. 5th Intl. Conf. on Knowledge Discovery and Data Mining, pages 53-62, 1999.

[5] M. Gomez-Rodriguez, J. Leskovec, and A. Krause. Inferring networks of diffusion and influence. In Proc. 16th ACM SIGKDD Conf. on Knowledge Discovery and Data Mining, pages 1019-1028, 2010.

[6] M. W. Koch and S. A. Mckenna. Distributed sensor fusion in water quality event detection. Journal of Water Resources Planning and Management, 137:10-19, 2011.

[7] A. Krause, J. Leskovec, C. Guestrin, J. VanBriesen, and C. Faloutsos. Efficient sensor placement optimization for securing large water distribution networks. Journal of Water Resources Planning and Management, 134(6):516-526, November 2008.

[8] M. Kulldorff. A spatial scan statistic. Communications in Statistics: Theory and Methods, 26(6):1481-1496, 1997.

[9] S. Myers and J. Leskovec. On the convexity of latent social network inference. In Advances in Neural Information Processing Systems 23, pages 1741-1749. 2010.

[10] D. B. Neill. Expectation-based scan statistics for monitoring spatial time series data. International Journal of Forecasting, 25:498-517, 2009.

[11] D. B. Neill. Fast subset scan for spatial pattern detection. Journal of the Royal Statistical Society (Series B: Statistical Methodology), 74(2):337-360, 2012.

[12] D. B. Neill, A. W. Moore, M. R. Sabhnani, and K. Daniel. Detection of emerging space-time clusters. In Proc. 11th ACM SIGKDD Conf. on Knowledge Discovery and Data Mining, 2005.

[13] S. Speakman and D. B. Neill. Fast graph scan for scalable detection of arbitrary connected clusters. In Proc. International Society for Disease Surveillance Annual Conference, 2010.

[14] R. Viswanathan and P. K. Varshney. Distributed detection with multiple sensors: Part I Fundamentals. Proceedings of the IEEE, pages 54-63, 1997. 